\title{
Las prácticas de extensión y la investigación universitaria sobre los Determinantes Sociales de la Salud: enfoques y reflexiones actuales
}

Roxana Prósperi

Docente investigadora de la Escuela Superior de Sanidad de la Facultad de Bioquímica y Ciencias Biológicas. Universidad Nacional del Litoral.
Universidad y salud /

Perspectivas

\section{Resumen}

Las intervenciones sobre los Determinantes Sociales de la Salud (DSS) se generan a partir de la identificación de inequidades y desigualdades sociales entendidas como problemas que afectan el bienestar y la calidad de vida de la población. Estos aspectos sintonizan y se retroalimentan a través de la promoción de líneas de investigación interdisciplinarias destinadas a conectar los factores individuales con las problemáticas estructurales que producen las inequidades en salud a nivel de políticas públicas.

Las prácticas de extensión, por su misma naturaleza, se hallan vinculadas con la pertinencia social del conocimiento que se genera en la universidad. En este trabajo nos proponemos reflexionar sobre las actividades de investigación y las construcciones de conocimiento que en la actualidad se consideran como condición de posibilidad para propiciar y enriquecer dichas prácticas.

Palabras clave

- determinantes sociales en salud

- investigación universitaria

- extensión

\section{Introducción}

El Informe de la Comisión de los Determinantes Sociales de la Salud (DSS) de la Organización Mundial de la Salud (OMS), 2008, posicionó en el debate mundial la importancia de las problemáticas sociales en la producción de las condiciones de enfermedad, muerte y atención a la salud. Son sus célebres antecedentes el Informe Lalonde (Canadá, 1974), a partir del cual se evidenció que los principales problemas de salud no están vinculados con la asistencia sanitaria sino con los hábitos de vida de la población, y el Informe Black (Reino Unido, 1980) que demostró las diferencias marcadas en el riesgo de morir entre las clases ocupacionales. Tomando lo que resulta de interés para este trabajo, dichos informes llaman la atención sobre la necesidad de desarrollar una actividad científica que se comprometa en su accionar con los problemas de salud originados por las condiciones de vida. Recapitulando lo señalado en estos informes, los DSS se pueden caracterizar conceptualmente de este modo:

"utilizamos el término desigualdad social en salud, para traducir del inglés inequity in health, es decir, la falta de equidad en la distribución de la enfermedad. Así pues, para que una diferencia o desigualdad en salud sea considerada un DSS, tienen que darse dos condiciones:

- que se la considere socialmente injusta,

- que sea potencialmente evitable (hay instrumentos para haberla evitado)" (Segura del Pozo, 2011:20-21).
La epidemiología social es una de las principales disciplinas que investiga los determinantes sociales de las distribuciones de la salud, la enfermedad y el bienestar de la población. Se pueden considerar sus tendencias teóricas actuales:

"la teoría psicosocial, que conceptualiza en torno al individuo (su comportamiento y sus hábitos) y que sostiene que los factores agudos y crónicos del ambiente social pueden volverse directamente patogénicos e inducir a comportamientos que dañan la salud; la teoría de la producción social de la enfermedad que sostiene que el ámbito económico-político en el que se gestan las desigualdades sociales, es la causa principal de las desigualdades en el campo de la salud; y la teoría ecosocial, que utiliza la categoría de "expresión biológica de la desigualdad social" para explicar, por ejemplo, las diversas formas de enfermar propias de la pobreza" (Moiso, 2007:166).

En América Latina, el desarrollo de la epidemiología crítica -cuyos antecedentes son la medicina social y la salud colectiva latinoamericana - propone una reformulación radical de las categorías epistemológicas y teóricas de la epidemiología convencional. Estudia las maneras en que los procesos históricos generales tienen manifestaciones particulares en la corporeidad humana y permiten comprender perfiles patológicos de grupos sociales. ${ }^{1}$

1) Sus principales impulsores son Jaime Breihl y Edmundo Granda

(Ecuador), Asa Cristina Laurell (México)

y Naomar Almeida Filho (Brasil). 


\section{6}

la epidemiología social es una de las principales disciplinas que investiga los determinantes sociales de las distribuciones de la salud, la enfermedad y el bienestar de la población
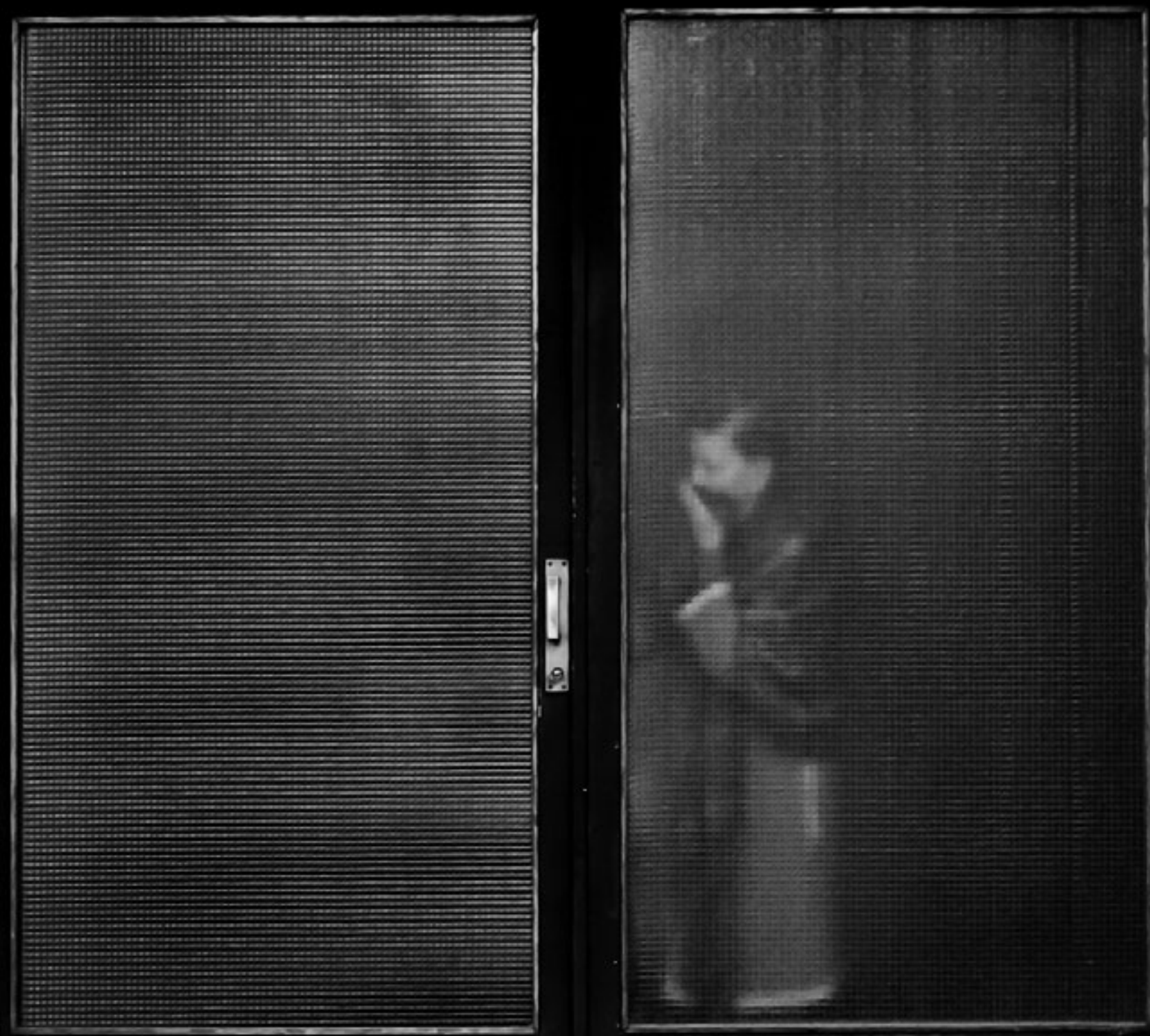
En el mismo año de la publicación del citado Informe de OMS, a la par del reconocimiento de su exhaustividad se plantearon puntos de discusión sobre algunas de sus concepciones. Con referencia a la producción de conocimiento, el Taller Latinoamericano de DSS, planteó:

"La postura de reunir evidencia sobre los factores sociales que generan desigualdades en salud, reproduce limitaciones del paradigma dominante en la epidemiología y la salud pública (Almeida, 2000; Breilh, 2003), pues fragmenta la realidad en factores, suponiendo que aislados mantienen su capacidad explicativa y son susceptibles de ser modificados" (López Arellano, Escudero, Dary Carmona, 2008:327).

Se propugna por un enfoque en el que la salud deje de ser considerada como tema específico de los profesionales de esa área para pasar a ser una cuestión social, política, histórica, económica y ética.

La UNL cuenta con un Programa de Extensión "Equidad en Salud", que se propone entre sus objetivos:

"Abordar de forma integral e interdisciplinaria los temasproblemas prioritarios identificados en el área de la salud con el fin de aportar posibles soluciones y/o caminos de superación de las situaciones diagnósticas detectadas" (UNL, 2006:10).

Una propuesta de esta magnitud refuerza el rol de la universidad en la cooperación con ámbitos sociales externos en la generación de conocimientos con pertinencia social y en la apropiación de los mismos por parte de los actores involucrados. En este trabajo, en primer lugar, expondremos algunos rasgos definitorios del paradigma dominante en la investigación en salud. En segundo lugar, nos proponemos reflexionar sobre las características de las actividades de investigación que se consideran como condición de posibilidad para propiciar y enriquecer el abordaje integral mencionado precedentemente, centrándonos en aquellas que actualmente pugnan por adquirir mayor protagonismo.

\section{El paradigma dominante en la investigación en salud} Dentro de la epidemiología convencional, aún predominante, los problemas a tratar se analizan como "factores" vinculados con las elecciones de un estilo de vida inadecuado, sin avanzar en el modo en que se ejerce la interacción de sus causas contribuyentes, desarticulando así los fenómenos de salud- enfermedad de los restantes procesos sociales en los que están inmersos. Según una expresión de uso muy extendido, estos últimos son considerados como "telón de fondo" de los fenómenos biomédicos.

Los criterios de validación del conocimiento científico en el área de la salud que da sustento al paradigma dominante provienen de: - Revisiones sistemáticas y metanálisis, principalmente de los resultados de ensayos clínicos controlados, que son considerados la "mejor evidencia disponible" (Sackett, Strauss, Richardson, Rosenberg y Haynes, 2000). ${ }^{2}$ Se ubica en un nivel de menor rango científico a otro tipo de diseños que no evalúan el resultado de intervenciones tecnológicas sino otros problemas de salud que obedecen a causas distintas. El mayor ejemplo al respecto es la investigación experimental financiada por la industria farmacéutica que, si bien es parte necesaria del sistema de salud, es también fuente de problemas e interrogantes sobre la accesibilidad, la pertinencia y la responsabilidad social en su aplicación y/o utilización y en la definición de las prioridades en atención sanitaria de la población.

- El tipo de conocimiento exigido en la actual "sociedad del conocimiento" (Drucker, 1993; Fuentes Ortega y Callejo Hernanz, 2007), que prioriza una formación basada en competencias entendidas como un "saber hacer" necesario para insertarse en el mundo del trabajo. La capacitación con alto nivel de especialización es un requisito para formar parte del mundo de la producción y del mercado y para la generación de capital y riqueza. Como puntuamos en otros textos (Brussino y Prósperi, 2011), si bien se pone el foco en aspectos de incuestionable valor y utilidad, su pretensión de instituirse como pensamiento hegemónico tiene múltiples consecuencias. La inversión en innovación e investigación, en consonancia con los requerimientos de las empresas y la competitividad económica, es un aspecto que define las prioridades de la agenda de investigación académica del mundo actual a través de las revistas internacionales de mayor prestigio:

"Un sistema de evaluación que busca legitimidad académica entendida en forma restringida, que está dispuesto a premiar la vinculación con la producción porque ésta goza de legitimidad en la economía del conocimiento, aunque lo que haya para premiar sea poco debido a la debilidad estructural de dicha vinculación, y que no considera siquiera premiar la investigación orientada a la inclusión social, conforma un sistema de señales intrínsecamente contradictorio" (Arocena y Sutz, 2009:112).

2) La Cochrane Collaboration (www.

altaveu.chpt.es/cochrane) es una de las

principales redes internacionales informati-

zadas que prepara y difunde las revisiones

sistemáticas y que se sostiene en un

sistema cerrado de pautas de validación

basadas en el método experimental. 
la epidemiología, además de desarrollar

los conceptos que le son propios como

disciplina, se ve en la necesidad de utilizar

nociones y conceptos que pertenecen a

otros campos del conocimiento

Vehiculizado históricamente a través de los organismos internacionales de financiamiento (Llomovate, 2006), este modelo perpetúa la concentración de la capacidad de producción del saber científico tecnológico del sistema capitalista mundializado. Los problemas propios de los sectores más postergados, que no pueden atribuirse meramente a los estilos de vida de sujetos individuales sino a consecuencias de políticas públicas, no ingresan fácilmente en dichas agendas ni en los estándares de investigación académica de alto nivel (Nairdorf, 2011). Sin embargo, la atención integral al fortalecimiento de la demanda, al desarrollo y a la inclusión social no pertenece sólo al ámbito de las decisiones políticas, como suele entenderse, sino que también requiere de la producción y uso del conocimiento científico.

\section{El estudio de los DSS}

La vinculación entre las ciencias sociales y las ciencias de la salud, con la consiguiente constitución de un nuevo campo teórico, surge con posterioridad a la Segunda Guerra Mundial, aportando un enfoque que se ocupa de la etiología social de las enfermedades y la aplicación del conocimiento social a los problemas de salud. Los determinantes que son considerados como "estructurales" son aquellos que se originan en un modelo político, económico y social que incide directamente en el acceso y distribución de la riqueza así como en las desigualdades en ingresos, educación y ocupación. Requiere elaboraciones teóricas explícitas sobre la sociedad y las condiciones de vida, que en conjunción permiten construir un pensamiento crítico para identificarlos, analizarlos y ayudar a solucionarlos.
Sin embargo, tal como expresa Almeida (1992), la epidemiología, además de desarrollar los conceptos que le son propios como disciplina, se ve en la necesidad de utilizar nociones y conceptos que pertenecen a otros campos del conocimiento. De este modo, muchos estudios presentan adecuadas validaciones de las variables dependientes epidemiológicas pero que se ponen en vinculación con variables independientes de otras disciplinas que no han pasado por una evaluación crítica de su validez teórica u operacional para su uso epidemiológico. Vale decir que "estarían tomando como implícitos cuadros conceptuales, detrás de sus variables independientes, pudiendo recibir críticas severas en el interior de las disciplinas de donde son originarios" (Almeida, 1999:39).

Ejemplo de esto es, tanto para investigaciones epidemiológicas como para las demás disciplinas del área de la salud, la utilización sin análisis de nociones como: "pobreza", "exclusión social", "vulnerabilidad", "calidad de vida", "desigualdad social”, "inequidad social", "nivel educativo", "clase social” y "posición socioeconómica". ${ }^{3}$ La mayoría de estas nociones es estudiada por las ciencias sociales a través de desarrollos exhaustivos $\mathrm{y}$, dado el nivel de complejidad de algunas, se hallan en un permanente estado de discusión acerca de los marcos conceptuales, las dimensiones de estudio y las formas de construcción de indicadores que se desprenden de ellas. Con relación a esto último, son ejemplos muy significativos los casos de "calidad de vida" (Saitén, 1993) y de "clase social". En este último, por ejemplo, su complejidad teórica proviene de que un sistema de clases es una jerarquización colectiva que no resulta solamente de las categorías 
de empleo, ya que la clase social determina las oportunidades de un individuo y también incluye las maneras de comportarse, los gustos, el lenguaje, las opiniones, las creencias éticas y religiosas. Tomando en cuenta la intensa asociación de la clase social con la mayoría de los factores relacionados con la salud, los marcos de referencia para abordar su estudio y las propuestas metodológicas para construir sus indicadores, se debaten en la actualidad en el mundo entre las escuelas neomarxista y neoweberiana (Regidor, 2001). Por la misma razón, resulta insoslayable incorporar esos aportes a las investigaciones en salud a través de la constitución de verdaderos grupos interdisciplinarios que, si bien no abundan en la práctica, son considerados la opción indispensable para comprender la naturaleza de problemas que responden a una compleja red de causas contribuyentes y que, además, se ubican en diferentes dimensiones (biológica/subjetiva/social, de la conducta objetiva/de las intenciones mentadas subjetivamente, macro y microsocial, etcétera).

\section{Investigación crítica en salud}

Las ciencias de la salud, a través de los métodos cuantitativos, abordan analíticamente a la enfermedad desmembrándola en variables que permiten enfocar y profundizar al máximo el objeto de estudio, explicando las regularidades de los fenómenos a través de asociaciones estadísticas entre dichas variables. La reflexión sobre sus límites constituye un lugar común en la literatura metodológica. Si bien la investigación en ciencias sociales bajo la orientación cualitativa ya contaba con antecedentes significativos, a partir de la década de 1970 tuvo un importante desarrollo en diferentes campos, particularmente en el que es propio de la salud-enfermedad, efectuando una recuperación de los métodos cualitativos a través de investigaciones que buscaron comprender la enfermedad en un sentido social que viniera a paliar o complementar el reduccionismo y las falencias de las explicaciones biologicistas y centradas en lo estadístico. ${ }^{4}$

Las orientaciones cuantitativa y cualitativa tienen un carácter insustituible, irreductible e incompleto (Bericat, 1998) y el interés para llevar a cabo una u otra debe basarse en el tipo de problema que se va a investigar y el punto de vista desde el que se va a enfocar.
Las metodologías cualitativas se proponen dar visibilidad a los elementos subjetivos que por su misma naturaleza no pueden ser traducidos en cantidades. Para comprender el sentido de las acciones concretas y la constitución del mundo social objetivo, es menester ahondar científicamente en esta dimensión humana. Atender a los aspectos cualitativos de la salud y la enfermedad supone la aceptación de que la realidad y sus problemáticas no se reducen a lo captable como fenómeno a través de la observación, sino que también es lo simbólico que requiere ser interpretado (Souza Minayo, 1995). Para esto, se basan en relatos personalizados o testimonios y construyen sus análisis dentro del marco de los grupos primarios de referencia de los sujetos estudiados, mostrando así la importancia de producir datos que estén debidamente contextualizados. A través de la complejidad de los relatos personales (Pujadas Muñoz, 1992), de las trayectorias vitales de los sujetos y de sus grupos primarios, se muestra que éstos son una individualidad irreductible, pero también activos representantes de un modelo normativo social.

Esta dualidad resulta de mucha importancia al momento de registrar datos significativos para la investigación científica. El concepto de "necesidades sentidas", de intenso uso en los campos educativos y de salud entre las décadas de 1940 y 1960, supone la existencia de necesidades definidas por el sujeto, diferenciándose de las observadas por el personal de las instituciones educativas y de salud, que son necesidades objetivas. El concepto reconoce la existencia de ambos puntos de vista y muestra la importancia de abordar ambos. Un sujeto o grupo, puede negar la presencia de situaciones que lo afectan negativamente, ya que:

- Si bien las reconoce, las refiere a su forma de vida expresando en gran medida las representaciones dominantes de la sociedad a la que pertenece, por ejemplo, justificando a través del relato determinadas situaciones o expresando las razones de su existencia de un modo convalidador.

- Puede no reconocer problemas graves que lo aquejan, porque por ejemplo determinadas formas de vida degradantes son naturalizadas y por tanto, no registradas como degradantes ni mucho menos como revertibles.

- Puede ocultarlos intencionalmente, por ejemplo cuando el miedo o la vergüenza operan como amenaza que impide expresarse libremente. 


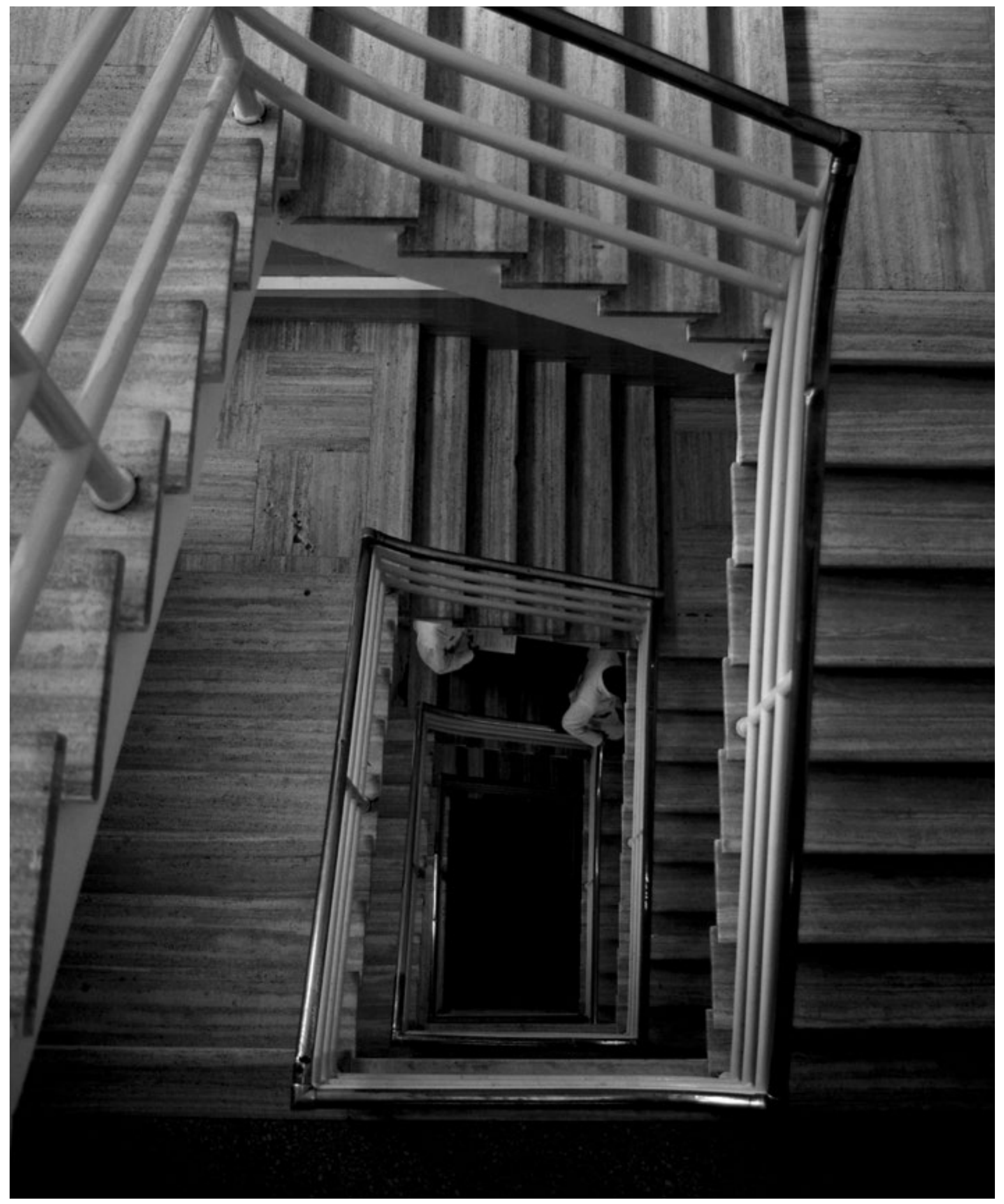


- Puede mentir, distorsionar, confundir, estigmatizar, con respecto a sí mismo y a los otros. Estas dificultades pueden afrontarse si se atiende a la necesidad de acceder a un nivel de comprensión que supere las disociaciones:

"En el nivel microsocial se desarrollan procesos que no pueden ser explicados en términos puntuales a partir del análisis macrosocial, así como la dimensión macro tiene una dinámica que no se puede comprender si se la observa exclusivamente a través del nivel microsociológico. De ahí que deberíamos pensar la realidad a partir de niveles articulados, de tal manera que aunque nos centremos en uno de los niveles reconozcamos que sólo la articulación nos permitiría obtener una lectura comprensiva de la problemática planteada. El reconocimiento de estas limitaciones puede operar como un control epistemológico de nuestros sesgos descriptivos, analíticos o interpretativos y de las persistentes tendencias a la extrapolación" (Menéndez, 2002:313).

Metodológicamente esto significa que el relato de los informantes es imprescindible, pero no habla por sí mismo, sino que la narrativa recopilada debe ser analizada en contacto con las determinaciones de su mundo de pertenencia para develar cuestiones que podrían estar ausentes en sus descripciones o estar presentes de un modo distorsionante. Atendiendo a estos niveles articulados, la aproximación crítica a lo social se produce cuando la investigación puede combinar la voz y el protagonismo de los sujetos individuales con la matriz de relaciones objetivas en que están insertos. Así, la investigación científica puede incidir directamente en el modo en que se identifican demandas sociales.

\section{Reflexiones finales}

Las actividades de investigación son condición de posibilidad para propiciar y enriquecer el abordaje integral y el aporte de soluciones a problemas relacionados con los DSS. Algunas de sus características principales que actualmente pugnan por adquirir mayor protagonismo son:

- El reconocimiento de los límites propios de las orientaciones cuantitativa y cualitativa de la investigación y la necesidad de una articulación entre ambas que propicie un nivel integrado en la compresión de los problemas. Para hacer mediciones y análisis del problema de los DSS, es necesario que haya sensibilización y formación en esta materia así como la construcción de sistemas de datos sobre inequidades sanitarias. Los afectados por estas inequidades no siempre se encuentran en situación de expresar su propia voz sobre un estado de cosas que se considera insatisfactorio. En algunos casos, la solución de los problemas denunciados requiere de la producción de nuevos conocimientos; en otros, es preciso construir esa demanda a través de indagaciones que identifiquen los problemas. En ambos casos se realizan esfuerzos para "colaborar a que el radar académico de los investigadores, que detecta problemas que están interesados en abordar, capture señales que vienen de la exclusión social" (Alzugaray, Mederos y Sutz, 2001:6).

A los fines de plantear programas o intervenciones, la superación de la fragmentación de la realidad en factores y la recuperación de la dimensión sociohistórica de la salud/enfermedad permiten formular recomendaciones que se centran en cada contexto particular.

- La constitución de equipos interdisciplinarios integrados por profesionales de la salud y de las ciencias sociales, ya que la expresión del vínculo universidad-sociedad requiere procesos de discusión conceptual en los que necesariamente están involucradas diferentes disciplinas para propiciar

- espacios de reflexión donde la universidad se interrogue a sí misma acerca del sentido de la formación académica y sus implicancias en el mundo actual (Vallaeys, De la Cruz y Sasia, 2009);

- estimaciones sobre el alcance del conocimiento socialmente útil que frecuentemente incide en un sector minoritario, que es el que detenta el capital;

- formas de comunicación que están llamadas a dejar de lado la unilateralidad de la mera transmisión, brindando la oportunidad a los grupos sociales de ser transformadores de sus propias relaciones con el mundo;

- propuestas de investigación que aporten a la inclusión y al desarrollo regional.

- El desarrollo de investigaciones que posibiliten un pensamiento crítico destinado a identificar y actuar frente a las formas de dominación y procesos de hegemonización presentes en las sociedades actuales y sus consecuencias para la salud (Kliksberg, 2011). Esto nos lleva a considerar la investigación no como mera representación del mundo sino como acción política responsable para proponer comprensiones que intervengan sobre las inequidades sociales. Se trata de contribuir, a través de investigaciones socialmente acordadas, a dar fundamento a decisiones legitimadas tanto teórica como éticamente, a identificar factores modificables y a reivindicar los intereses políticos regionales. Igualmente importante es que formen parte de esta discusión el sistema de prioridades que determina los temas que vale la pena investigar y los criterios con que se evalúa la producción científica académica.

- Inclusión de desarrollos teóricos explícitos sobre la dinámica social. En palabras de Briceño León:

"Es realmente un campo donde es necesario aún mucho esfuerzo teórico que sea capaz de construir teorías que permitan agrupar y darle coherencia y sentido a las experiencias de investigación e intervención" (2003:42). 


\section{Bibliografía}

Almeida Filho, N. (1992). Epidemiología sin números. OPS, Serie Paltex №28, Washington DC

Alzugaray S., Mederos L. y Sutz, J. (s/f). "La investigación científica contribuyendo a la inclusión social". Revista Ciencia, Tecnología y Sociedad, vol. 6, № 17.

Arocena, R. y Sutz, J. (2009). "Sistemas de innovación e inclusión social". Pensamiento Iberoamericano No 5. Disponible en: http://www. pensamientoiberoamericano.org/sumarios/5/innovacion-y-conocimiento/

Bericat, E. (1998). La integración de los métodos cuantitativo y cualitativo en la investigación social. Significado y medida. Barcelona, Ariel.

Brussino, S. y Prósperi, R. (2011). "Investigación universitaria en salud. Criterios de validación y ética profesional". Revista Redbioética/UNESCO, 2, 1(3), 9, enerojunio. Disponible en: http://www.unesco.org.uy/shs/red-bioetica/fileadmin/shs/ redbioetica/revista_3/revista3.pdf

Briceño León, R. (2003). "Las ciencias sociales y la salud: un diverso y mutante campo teórico". Ciencia \& Salud Colectiva, vol. 8, № 1:33-45.

De Souza Minayo, M. C. (1995). El desafío del conocimiento. Investigación cualitativa en salud. Buenos Aires, Lugar Editorial.

Drucker, P. (1999). La Sociedad Postcapitalista. Buenos Aires, Sudamericana. Elizondo Montemayor, L.; Ayala Aguirre, F. (2007). "El equilibrio de la enseñanza y la investigación en países latinoamericanos". Revista Iberoamericana de Educación/ OEl, 44/4:7. Disponible en: http://www.rieoei.org/deloslectores/1913Elizondo.pdf Fuentes Ortega, J. B. y Callejo Hernanz, M. J. (2007). "En torno a la idea de "sociedad del conocimiento". Cuaderno de materiales. Filosofía y Ciencias Humanas, № 17. Madrid. Disponible en http://www.filosofia.net/materiales/num/num17/Critilou.htm Kliksberg, B. (2011). "Estrategias metodológicas para promover la participación social en la definición e implantación de políticas públicas de combate a las inequidades en salud". Documento preparado a partir de la Conferencia Mundia sobre Determinantes Sociales de la Salud. Rio de Janeiro. Disponible en: http:// cmdss2011.org/site/wp-content/uploads/2011/07/Participaci\%C3\%B3n-Social_Bernardo-Kliksberg.pdf

Llomovate, S. (2000). "Para una crítica del modelo de la triple hélice: Universidad, Empresa y Estado". En Llomovate, S.; Juarros, F.; Nairdorf, J. y Guelman, A. La vinculación universidad-empresa: miradas críticas desde la universidad pública. Buenos Aires, Miño y Dávila.
López Arellano, O.; Escudero, C.; Dary Carmona, L. (2008). "Los determinantes sociales de la salud. Una perspectiva desde el Taller Latinoamericano de Determinantes Sociales de la Salud, ALAMES". Medicina Social, vol. 3, № 4.

Menéndez, E. (2002). La parte negada de la cultura. Relativismo, diferencias y racismo. Barcelona, Ediciones Bellaterra.

Moiso, A. (2007). "Determinantes de la salud". En: Barragan, H.L., (Ed). Fundamentos de la Salud Pública, pp. 161-189. Argentina, La Plata: Universidad Nacional de La Plata, Editorial EDULP.

Nairdorf, J. (2011). "Criterios de relevancia y pertinencia de la investigación universitaria y su traducción en forma de prioridades". Revista de la Asociación de Sociología de la Educación, vol. 4, № 4. Buenos Aires.

Pujadas Muñoz, J. (1992). "El método biográfico: El uso de las historias de vida en ciencias sociales". Cuadernos Metodológicos, N⒌ Centro Investigaciones Sociológicas (CIS) Madrid.

Sackett, L.; Strauss S.; Richardson, W.; Rosenberg, W. y Haynes, R. (2000). Evidence-Based Medicine. How to Practice and teach EBM. New York, Churchill Livingstone.

Saitén, M. L. (1993). "Indicadores de calidad de vida. Un sistema de medición aplicado al País Vasco". Cuadernos Metodológicos, № 133. Centro Investigaciones Sociológicas (CIS) Madrid.

Segura del Pozo, H. (2011). Desigualdades sociales en salud: conceptos, estudios e intervenciones (1980-2010). Archivo pdf disponible en: http://www. medicina. unal.edu.co/ADMON_CMS/ADJUNTOS/20120924_201909_Desigualdades\%20 Sociales\%20en\%20Salud_con-est-int-7_dic\%202011\%20(2).pdf

Regidor, E. (2001). "La clasificación de clase social de Goldthorp: marco de referencia para la propuesta de medición de clase social del grupo de trabajo de la Sociedad Española de Epidemiología". Revista Española de Salud Pública, vol. 75, №1. Madrid. Vallaeys, F.; De la Cruz, C.; Sasia, P. M. (2009). Responsabilidad social universitaria. Manual de primeros pasos. México DF, McGraw Hill Interamericana. Disponible en: http://rseonline.com.ar/2010/04/manual-de-responsabilidad-social-universitaria-rsu-bid/

Universidad Nacional del Litoral (2006). Programa de Extensión "Equidad en Salud". Disponible en: http://www.unl.edu.ar/categories/view/equidad_en_salud\#.U_pwkcV5Pil 